\title{
Micro-Raman Characterization of: Phase Formation and Thermal Stability of Nickel Silicide Thin Films.
}

\author{
G. Conti, C.Lazik, and Y. Uritsky \\ Defect and Thin Film Laboratory, Applied Materials Inc, 3100 Santa Clara, Ca 95054,USA
}

Fast and nondestructive characterization techniques are crucially important in developing new materials. Micro-Raman Spectroscopy is a powerful and versatile tool. Its capabilities range from structural identification of molecules to identification of crystalline structure of materials, to stress determination of STI (Shallow Trench Isolation) trenches, and SiGe structures. This power and versatility has led to its progressively more extensive applications to semiconductor industry.

In this paper we present the characterization of phase formation and thermal stability of silicide film by Raman analysis.

Silicides play an important role in decreasing RC delay time of circuits and thus in increasing performance and speed of devices through reduction of sheet, parasitic, contact, and interconnect resistances within polycrystalline silicon gate and source/drain regions. Because of its low resistivity, low formation temperature, and low silicon consumption, nickel mono-silicide (NiSi) is a promising candidate in sub-50nm CMOS technology [1]. The thermal stability of NiSi thin film and the phase transformation from $\mathrm{NiSi}$ to $\mathrm{NiSi}_{2}$ are of crucial importance for understanding the application of nickel silicides. In the $\mathrm{Ni}-\mathrm{Si}$ binary system, $\mathrm{Ni}$ diffuses into the silicon substrate to form the unstable and high resistivity phase $\mathrm{Ni}_{2} \mathrm{Si}$ at about $200{ }^{\circ} \mathrm{C}$. The preferred low resistivity NiSi phase starts to nucleate at $350{ }^{\circ} \mathrm{C}$, and another high resistivity phase $\mathrm{NiSi}_{2}$ nucleates above $750{ }^{\circ} \mathrm{C}$, which should be avoided in device fabrication. Micro-Raman spectroscopy is a suitable technique for characterizing the phase formation and the thermal stability of nickel silicide films [2-3]. Compared with traditional characterization techniques, Micro-Raman possesses a number of advantages: it is fast, real-time, non-contact and nondestructive, does not require special sample preparation; furthermore, it possesses a good spatial resolution of about $0.5 \mu \mathrm{m}$.

Raman measurements in backscattering configuration were performed on the samples as reported in table I, to identify the silicide phase formation (Fig. 1), the film thickness, and the local grain orientation (Fig. 2). The Raman results were compared to TEM; XRR and AFM results.

All the analyzed samples show the typical Raman peaks of the NiSi phase. However, the Raman spectrum of the samples post treated at $\mathrm{T}=700 \mathrm{C}$ indicates that $\mathrm{NiSi}_{2}$ phase starts to nucleate. The Raman results agree with the sheet resistance measurements. The sheet resistance is increasing from $4.5 \Omega /$ sq. to $6.5 \Omega /$ sq. for the samples post annealed at $\mathrm{T}=700^{\circ} \mathrm{C}$.

Transport properties are strongly related to the film microstructure and local orientation. MicroRaman has been successfully applied to characterize the epitaxy degree of various films. Crystalline grains differently oriented relative to the laser light give rise to different Raman peaks, which can be employed to obtain qualitative information of the degree of film epitaxy at the micrometer scale. Compared with XRD which probes the average film orientation at $\mathrm{mm}$ to $\mathrm{cm}$ scale, micro-Raman probes the film local orientation at the micrometer scale. Fig. 2 presents 
a series of Raman spectra of NiSi film before and after RTP annealing at $700{ }^{\circ} \mathrm{C}$ indicating that the grains of the NiSi have a variety of orientations at the micrometer scale. AFM analysis of these samples shows a grained structure in agreement with the orientation values obtained by Raman

Film characterization in terms of thickness, chemical composition, crystalline structure and crystallite orientation is very important for the development of future devices and for the control of the fabrication processes. In this paper analyzing nickel silicide films, we were able to identify the crystalline phase, the degree of orientation of the crystallites, and the overall thickness of the films. We believe that micro-Raman spectroscopy can be a valuable metrology technique in thin film characterization in the micrometer scale.

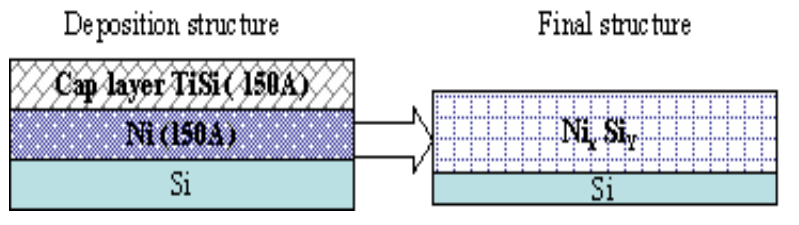

$\begin{array}{ccccc}\begin{array}{c}\text { Deposition } \\ \text { Structure }\end{array} & \begin{array}{c}\text { Deposition } \\ \text { Temperature }\end{array} & \text { 1st RTA } & \begin{array}{c}\text { TiN and } \\ \text { Un-reacted Ni }\end{array} & \begin{array}{c}\text { 2nd RTA } \\ \text { (700C) }\end{array} \\ \text { TiN150A/Ni150A } & \text { RT } & \text { 400C } & \text { Stripped } & \text { No } \\ \text { TiN150A/Ni150A } & 300 \mathrm{C} & 400 \mathrm{C} & \text { Stripped } & \text { No } \\ \text { TiN150A/Ni150A } & \text { RT } & \text { 400C } & \text { Stripped } & \text { Yes } \\ \text { TiN150A/Ni150A } & 300 \mathrm{C} & \text { 400C } & \text { Stripped } & \text { Yes }\end{array}$

Table I : Nickel Silicide samples analyzed by Raman.

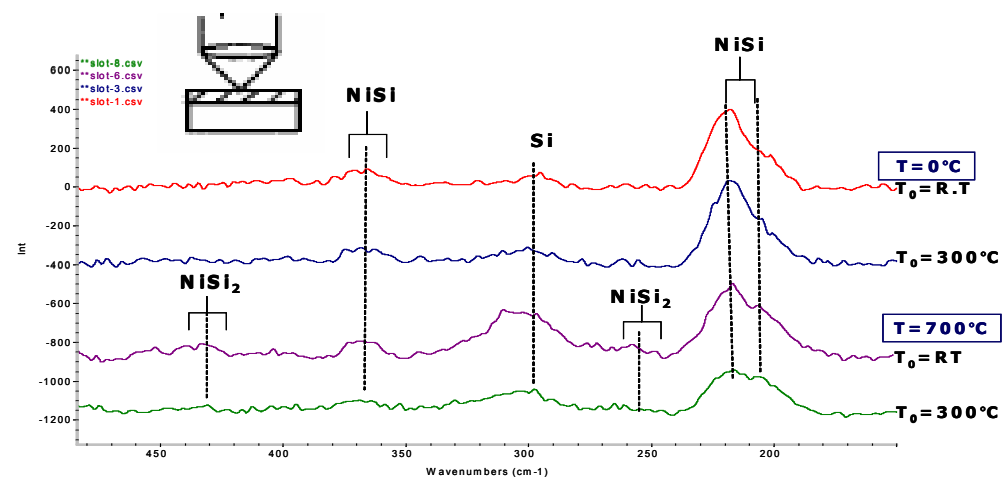

Fig.1 Raman spectrum shows different peaks for each NiSi phase.
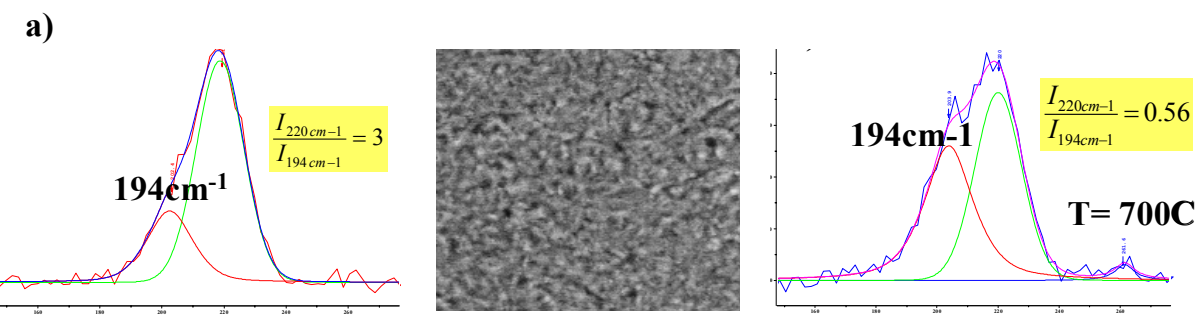

Fig.2 NiSi deposited at a) $\mathrm{T}=300 \mathrm{C}, \mathrm{b}) \mathrm{T}=700 \mathrm{C}$. The relative intensity ratio between the two peaks of the NiSi phase at $214 \mathrm{~cm}^{-1}$ and $195 \mathrm{~cm}^{-1}$ varies from 3.03 to 0.56 , indicating that the grains of the NiSi have a variety of orientations at the micrometer scale. AFM roughness measurements agree with Raman results.

\section{References}

[1] S. P. Murarka, Silicides for VLSI Applications Academic, New York, 1983

[2] P. J. Codelia, F. Adar, and Y. S. Liu, Appl. Phys. Lett. 45, 1076 , 1985.

[3] F. F. Zhao, at al., J. Vac. Sci. Technol. B, 862, 2003. 